\title{
Observational Study of the Tunisian Diabetic Patients' Profile during the Fasting of the Holy Month of Ramadan
}

\author{
Fatma Mnif ${ }^{1 *}$, Claude Ben Slama ${ }^{2}$, Larbi Chaieb ${ }^{3}$, Samira Blouza ${ }^{2}$, Mohamed Hsairi ${ }^{4}$, Mohamed Abid $^{1}$ \\ ${ }^{1}$ Department of endocrinology, CHU Hedi Chaker, Sfax, Tunisia \\ ${ }^{2}$ Institute of nutrition, Tunis, Tunisia \\ ${ }^{3}$ Department of endocrinology, CHU Farhat Hached,Sousse, Tunisia \\ ${ }^{4}$ Departement of Epidemiology, Salah Azaiez Institute Tunis, Tunisia
}

Received: July 15, 2016; Accepted: August 29, 2016; Published: August 30, 2016

*Corresponding author: Fatma Mnif, Department of Endocrinology, CHU Hedi Chaker, Sfax, Tunisia, Tel: +216-74 244 221; E-mail: fatmamnif05@yahoo.fr

\section{Abstract}

Aims: To analyze the profile of patients with Type 1 Diabete Mellitus (T1DM) and Type 2 Diabetes Mellitus (T2DM) fasting during Ramadan.

Methods: Descriptive observational epidemiological study in T1DM and T2DM diabetes treated by insulin and or oral antidiabetic drugs, older than 20 years old and who made their own decision to fast. Patients were recruited 1 to 30 days before Ramadan and followed-up up to 30 to 40 days after.

Results: 533 patients were included, 29 (5.4\%) were T1DM. The median age was 43.5 years for type 1 and 54.6 years for type2. The mean duration of diabetes was 8.47 years. $32 \%$ of type 2 diabetes were treated with insulin. Seven percent of type 2 patients stopped fasting at mid-Ramadan and six percent at the end of Ramadan. In T1DM $(11,1 \%$ and $0 \%)$ stopped fasting at mid-Ramadan and at the end of Ramadan respectively. The main cause for stopping fasting was hypoglycemia. The number of hypoglycemia in type 2 remained stable between before Ramadan vs. mid-Ramadan ( 0.2 vs 0.3 ). However it decreased significantly to 0.1 at the end of Ramadan. No significant changes were noticed for type 1 . We noted no increase of severe and nocturnal hypoglycemia. In the whole population, $27.6 \%$ had normal blood glucose $\leq 1.26 \mathrm{~g}$ / l before Ramadan vs $32.4 \%$ at mid Ramadan, and $35.9 \%$ at the end of Ramadan ( $p=0.008$ ). No significant difference for A1c between before Ramadan and at the end of the study. We noted a change of lifestyle during this month with a tendency to the reduction of the physical-activity and an increase in liquid ingestion at the beginning of Ramadan. Calories and carbohydrate intake were both lower during the 2 nd half of Ramadan for type 2 patients vs the first half. Weight remained stable during Ramadan and throughout the study. Approximately $24 \%$ of the patients adapted their treatment at the beginning of Ramadan mainly by dose ranging decrease.

Conclusion: Glycemic control was acceptable during Ramadan due to a change in lifestyle and attitudes of patients with regards to fasting. Hypoglycemia episodes increased during the first half and decreased significantly during the second half of Ramadan.

Keywords: Fasting; Type 1 diabetes mellitus; Type 2 diabetes mellitus; Ramadan

\section{Introduction}

Fasting Ramadan is a pillar of Islam. Healthy adult Muslim must refrain from drinking and eating from dawn to sunset of everyday of this month. However there are no restriction on food or fluid intake between sunset and dawn. Sick persons, travelers and pregnant women are exempted from this duty [1]. In fact, Muslims with diabetes and other chronic diseases are exempted from fasting, where fasting may lead to harmful consequences. Nevertheless, many patients insist on participating in Ramadan fasting [1]. It is estimated that around 40 to 50 million of individuals with diabetes worldwide fast during Ramadan [1,2]. The population based-Epidemiology Of Diabetes And Ramadan 1422/2001 (EPIDIAR) study conducted in13 Islamic countries revealed that $43 \%$ of patients with type 1 diabetes and $79 \%$ of patients with type 2 diabetes fast during Ramadan [1,2].

Since the publication of the EPIDIAR study in 2004, several reports on the safety, benefits and challenges of fasting in diabetes subjects have been published.

The aim of the current study is to identify the profile of type 1 and 2 diabetic patients deciding to fast during Ramadan. Secondly, to identify the current pattern of care of type 1 and type 2 diabetic patients by their treating physicians

Thirdly, to assess the outcomes of these patients, calories and liquid intake and the medical metabolic disorders occurring during the fasting period compared to non-fasting period

\section{Patients and Methods}

\section{Subjects}

From 21-7-2009 to 31-8-2009, 533 Tunisian patients were recruited from 54 centers. Patientswilling to fast during Ramadan 2009 for a period of 30 days (from 22 august until 21 September 2009), with diabetes mellitus type 1 or 2 diagnosed since more than 3 months, and age superior or equal to twenty 
years were included to this study. These patients were treated by either insulin or Oral Anti-Diabetic Drug (OAD) or both. Pregnant and nursing women as well as those over the age of procreation without taking contraceptive pills were excluded from this study. Besides, patients who refused the consent of the use of the data or those who participated simultaneously in another clinical study.

Recruited patients were followed according to an observational survey during around one hundred days. This survey consists of three periods, first, the inclusion period, planned one day to thirty days before the beginning of Ramadan (Visit 1). The second period is defined between 12 and 18 days after the beginning of Ramadan (visit 2). The last period was the period of "follow-up" consisting of two visits, one three to seven days after the end of Ramadan (visit 3 ) and another 30 to 40 days after the end of Ramadan (visit 4).

During V1, the following information was collected: age, sex, history of diabetes and related complications, weight, height, blood pressure and medication use; last measure of $\mathrm{HbA1c}$, fasting blood glucose, total cholesterol, low density lipoprotein cholesterol, high density lipoprotein cholesterol, triglycerides and the level of creatinine was recorded.

Estimation of previous Ramadan evolution was made (duration of fast, complications)

At each visit the occurrence of hypoglycemia (blood glucose $\leq 0.5 \mathrm{~g} / \mathrm{l}$ ), during the night or the day, severe or not) weight changes and blood sugar control were recorded

The data was collected via structured interview supported by a questionnaire .The objective of the interview was to gather socio-demographic data, days of fasting, the reason of interrupting the fast, lifestyle changing during Ramadan, frequency of hypoglycemia and compliance to the dietary instructions.

\section{Dietary intake}

T2DM patients recorded in an agenda, their daily dietary intake for three days of the same week chosen for each period of the study.

The dietary inquiry estimated the daily hydric and caloric intake, and mainly the glucose contributions. Thus, the patient answered on his caloric intakes on the basis of a pre-established list of meals, sweets and drinks. The patient selected the taken food, and specify the quantity according to the reference volume indicated on the questionnaire, by a number or an image.

\section{Statistical Analysis}

Data analysis included: The verification of the respect of inclusion and exclusion criteria, the description of the variables to the study relative to socio-demographic and economic history of the patients, medical and surgical history, prescribed treatments, data of the clinical exam and to results of complementary exams, the analysis of the evolution of data relative to clinical exam, biological complementary exams and ECG, observance of diet and lifestyle counseling, occurrence of intercurrent events including hypoglycemic episodes, and modalities of care
The comparison of percentages was done by the Chi square test, the Fisher exact test and the Mac Nemar test. The comparison of means was done by the Student test and by the analysis of variance test for the repeated measures.

All the statistical tests are bilateral with a risk $\alpha$ equal to five percent. The data will be captured by Epi info software and analyzed by STATA $^{\odot}$ software.

\section{Results}

\section{Baseline clinical characteristics}

Socio-demographic and economic characteristics (table 1): The present study included five hundred and thirty three patients of whom 29 where diabetic type 1 (5.4\%). Concerning the gender, $61.5 \%$ of the population is male. One third of them is aged 60 years or more and the rest is between 40 and 59 . The main age of type 1 diabetic patients is $43.5 \pm 17.3$ years and for type 2 patients, it is $54.6 \pm 9.8 .27 .6 \%$ of T1DM vs three percent of T2DM are single. Less than $8 \%$ consider having an unsatisfied socio-economic level, and $94 \%$ live in urban area. Most of the included patients have a medical insurance (95\%).

Approximately ninety percent of the participants fasted previously without interruption, $16.5 \%$ have presented complications and $2.4 \%$ had never fast. Among patients only $15.4 \%$ reported episodes of hypoglycemia during the previous month of Ramadan (14.1\% for T2DM and 37.9\% for T1DM respectively)

Medical history of diabetes: T2DM was frequently discovered within a systematic exam $(77.8 \%$ of the cases vs $48.3 \%$ for T1DM), or through complications $(16.9 \%$ for T2DM and $34.5 \%$ for T1DM).The mean diabetes duration is $8.5 \pm 7.1$ years for T2DM, distribute as follow: $<5$ years for $37.1 \%$ of patients, between five and nine years for $26,0 \%$ of patients, between 10 and 19 years for $28.0 \%$ of patients and $>20$ years for $8,9 \%$ of them.

The mean age of T2DM discovery is $45.9 \pm 9.3$ years vs 36.3 \pm 12.7 years for T2DM. In the same population, main associated diseases are hypertension (45.6\% of type 2 patients' vs $27.6 \%$ of T1DM $p<0.05)$, dyslipidemia $(30.2 \%)$ and retinopathy (10.1\%). Almost $17.3 \%$ of T2DM vs $24.1 \%$ for T1DM are smokers (cigarettes and/or narghile). Around $54 \%$ of patients practice sport, $69 \%$ of them follow a diet and $49.7 \%$ of them practice selfmonitoring blood glucose.

\section{Physical exam and glycemic control of the patients at V1}

The majority of patients have an optimal / normal blood pressure $(40.1 \%$ of T2DM vs $58.6 \%$ of T1DM) or a high normal blood pressure $(29.4 \%$ vs $20.7 \%$ respectively). high systolic blood pressure was noted in $18.6 \%$. The mean weight is $79.5 \pm$ $13.5 \mathrm{~kg}$ in T2DM and $77.0 \pm 14.1 \mathrm{~kg}$ in T1DM. This leads to a mean of BMI at $28.9 \pm 5.7 \mathrm{~kg} / \mathrm{m}^{2}$ and $27.4 \pm 5.6 \mathrm{~kg} / \mathrm{m}^{2}$ respectively.

Blood glucose values before the inclusion were normal in $27.1 \%$ of type 2 diabetes patients $(34.5 \%$ for type 1 ) and $29.7 \%$ of T2DM had normal glycate himoglobine (HbA1c) (16.7\% for type 1). 
Table 1:

\begin{tabular}{|c|c|c|c|c|c|c|c|}
\hline \multicolumn{3}{|c|}{ T1DM } & \multicolumn{2}{|l|}{ T2DM } & \multicolumn{2}{|l|}{ Total } & $\mathrm{p}$ \\
\hline $\operatorname{sex}$ & $\mathbf{n}$ & $\%$ & $\mathbf{n}$ & $\%$ & n & $\%$ & \multirow{3}{*}{0,74} \\
\hline male & 17 & 58,6 & 310 & 61,6 & 327 & $61,5 \%$ & \\
\hline female & 12 & 41,4 & 193 & 38,4 & 205 & $38,5 \%$ & \\
\hline \multicolumn{3}{|l|}{ Missing data $=1$} & \multicolumn{4}{|c|}{ Missing data $=1$} & \\
\hline AGE & $\mathbf{n}$ & $\%$ & $\mathbf{n}$ & $\%$ & n & $\%$ & \multirow{7}{*}{$<0,001$} \\
\hline $20-29$ & 11 & 37,9 & 4 & 0,8 & 15 & $2,9 \%$ & \\
\hline $30-39$ & 2 & 6,9 & 23 & 4,7 & 25 & $4,9 \%$ & \\
\hline $40-49$ & 3 & 10,3 & 123 & 25,3 & 126 & $24,5 \%$ & \\
\hline $50-59$ & 8 & 27,6 & 188 & 38,7 & 196 & $38,1 \%$ & \\
\hline $60-69$ & 3 & 10,3 & 119 & 24,5 & 122 & $23,7 \%$ & \\
\hline $70+$ & 2 & 6,9 & 29 & 6,0 & 31 & $6,0 \%$ & \\
\hline \multicolumn{3}{|l|}{ Missing data $=18$} & \multicolumn{4}{|c|}{ Missing Data $=18$} & \\
\hline & $\mathbf{n}$ & $\%$ & $\mathbf{n}$ & $\%$ & n & $\%$ & \multirow{5}{*}{$<0,001$} \\
\hline married & 18 & 62,1 & 466 & 92,6 & 484 & $91,1 \%$ & \\
\hline single & 8 & 27,6 & 15 & 3,0 & 23 & $4,3 \%$ & \\
\hline divorced & 3 & 10,3 & 2 & 0,4 & 5 & $0,9 \%$ & \\
\hline widower & 0 & 0.0 & 20 & 4,0 & 20 & $3,8 \%$ & \\
\hline \multicolumn{3}{|l|}{ Missing Data $=1$} & \multicolumn{4}{|c|}{ Missing Data = 1} & \\
\hline residence & $\mathbf{n}$ & $\%$ & $\mathbf{n}$ & $\%$ & n & $\%$ & \multirow{3}{*}{0,33} \\
\hline urbain & 25 & 89,3 & 460 & 93,9 & 485 & $93,6 \%$ & \\
\hline rural & 3 & 10,7 & 30 & 6,1 & 33 & $6,4 \%$ & \\
\hline \multicolumn{2}{|l|}{ Missing data $=1$} & \multicolumn{2}{|c|}{ Missing data $=14$} & \multicolumn{2}{|c|}{ Missing data $=15$} & & \\
\hline Socio-economic level & $\mathbf{n}$ & $\%$ & $\mathbf{n}$ & $\%$ & n & $\%$ & \multirow[b]{5}{*}{0,72} \\
\hline very satisfactory & 7 & 24,1 & 96 & 19,2 & 103 & $19,5 \%$ & \\
\hline satisfactory & 19 & 65,5 & 364 & 73,0 & 383 & $72,5 \%$ & \\
\hline non really satisfactory & 3 & 10,3 & 34 & 6,8 & 37 & $7,0 \%$ & \\
\hline unsatisfactory & 0 & 0.0 & 5 & 1,0 & 5 & $0,9 \%$ & \\
\hline \multicolumn{5}{|l|}{ Missing Data= 5} & \multicolumn{2}{|c|}{ Missing Data $=5$} & \\
\hline social Insurance & $\mathbf{n}$ & $\%$ & n & $\%$ & n & $\%$ & \multirow{3}{*}{0,74} \\
\hline yes & 24 & 96,0 & 430 & 94,5 & 454 & $94,6 \%$ & \\
\hline no & 1 & 4,0 & 25 & 5,5 & 26 & $5,4 \%$ & \\
\hline \multicolumn{3}{|l|}{ Missing data $=4$} & \multicolumn{2}{|c|}{ Missing data $=49$} & \multicolumn{3}{|c|}{ Missing data $=53$} \\
\hline Type of diabetes & Variable & $\mathrm{Nb}$ & Minimum & Maximum & Mean & Ecart-type & Median \\
\hline T1DM & AGE: & 29 & 20,0 & 78,0 & 43,5 & 17,3 & 42,0 \\
\hline T2DM & AGE: & 486 & 23,0 & 82,0 & 54,6 & 9,8 & 55,0 \\
\hline
\end{tabular}

Anti-diabetic therapy: Almost $68 \%$ of T2DM patients are treated with OAD alone $(33.9 \%$ as a bi-therapy, $24.5 \%$ as a mono-therapy) In twenty six percent of case insulin is given in association with $\mathrm{OAD}$ and $5.8 \%$ patients receive insulin as mono-therapy. Globally $52 \%$ of the patients received long-acting analogue insulin.

The main OAD prescribed are biguanide (44.6\%), sulfonylurea
(38.9\%), thiazolidinedione (8.7\%), alpha-glucosidase inhibitors (4.6\%) and glinides (3.1\%). In $69 \%$ of cases, concomitant treatments are prescribed (mainly anti-hypertensive, antiplatelet therapy, and lipid- regulating drug therapy).

Ramadan 2008 data: During Ramadan 2008, 88.8\% of types 2 and $72.4 \%$ of type 1 patients have previously fasted without interruption (respectively $9.2 \%$ and $17.2 \%$ have fasted 
with interruption). Sixteen percent (type 2) and $25 \%$ (type 1) reported complications during their previous fast and $2.4 \%$ had never fast. During previous months of Ramadan, globally $15.4 \%$ (14.1\% of type 2 and $37.9 \%$ of type 1 patients) reported hypoglycemia. Furthermore, the mean number of symptomatic hypoglycemia episodes per week was $0.2 \pm 0.8$ episode, no severe episode was brought back.

Evolution: The number of type 2 patients who had filled their patient agenda slightly decreased during the second half of Ramadan from $95.7 \%$ in V2 to $92.6 \%$ in V4 ( $p=0.07)$. The principal reasons of non-filling the agenda are the forgetfulness or the lack of glucometer.

Nine percent of patients didn't attend the V2, $17.6 \%$ of patients didn't attend the V3 and 15.8\% the V4. For type 2 diabetes patients, the mean systolic blood pressure between V1 and V2 decreased from $130.2 \pm 13,5 \mathrm{mmHg}$ to $127.5 \pm 13.1 \mathrm{mmHg}$. During V3 and V4, the systolic pressure increased to reach the level registered during V1. The same trend was observed for the diastolic blood pressure $(p=0.12)$.

At the beginning of the holy month of Ramadan, the proportion of T2DM patients practicing sport diminished from $53.7 \%$ in V1 to $45 \%$ in $\mathrm{V} 2(p<0.01)$.

Diet was abandoned in the end of Ramadan so that the proportion of type 2 patients observing diet decreased from $74.1 \%$ in $\mathrm{V} 2$ to $70.5 \%$ in $\mathrm{V} 4(p<0.05)$.

Interruption of fast was noted in $7.6 \%$ of type 2 patients at $\mathrm{V} 2$ and $6.8 \%$ at $\mathrm{V} 3$. The main reasons of fasting interruption are related to the occurrence of hypoglycemic episodes.

For type 1 patients, interruption occurred in $11.1 \%$ at V2 and $0 \%$ at V3 .Adaptation of treatment occurred mainly at the beginning of Ramadan, at V2, 76.7\% of T2DM patients maintain the same treatment. This proportion increased at V3 to $85.7 \%$ (V2 vs. V3 $p<0.01$ ). There's no significant variation between V3 and V4 $(p=0.59)$.

Hypoglycemia episodes: The percentage of type 2 patients presented hypoglycemic events significantly decreased (from $14.8 \%$ in V2 to $7.7 \%$ in V3 and $6.9 \%$ in V4 $p<0.01$ ).

In this population, the mean number of symptomatic hypoglycemic episodes increased from 0.2 episode in V1 to 0.3 in V2 and then stabilized at 0.1 episode in V3 and V4. However, no severe episode ( need hospitalisation) was reported.

For type 1 diabetes, no significant variation neither in percentage of patients nor in number of episode of hypoglycemia was noticed, because of a too small sample size.

Impact of fasting on blood glucose and HbA1c: In type 2 diabetes, the proportion of blood glucose values $<1.26 \mathrm{~g} / \mathrm{l}$ passed from $27.1 \%$ at $\mathrm{V} 1$, to $33.5 \%$ at $\mathrm{V} 2$, to $35.0 \%$ at $\mathrm{V} 3$ and then to $30.0 \%$ at $\mathrm{V} 4$ (V1 vs. V2 $p<0.001$ ).

No significant change in mean value of HbA1c between $\mathrm{V} 1$ (8.2\%) and V4 (8.1\%).
Impact of fasting on patients weight: The weight of T2DM patients increased in V4 $(79.5 \pm 13.5 \mathrm{~kg}$ in V1 vs $80.3 \pm 12.5 \mathrm{~kg}$ in V4; $p=0.008$ ). However, no weight variation occurred in type 1 patients group.

\section{Impact of fasting on nutriment intakes}

\section{Descriptive analysis of the dietary survey (table 2)}

Fluid Intake of drinking water: Volumes of drinks were higher in the month of Ramadhan compared to the period after Ramadhan. The average decreased from $1972.13 \pm 741.29 \mathrm{ml} / \mathrm{d}$ at V2; $1819.74 \pm 737.38 \mathrm{ml} / \mathrm{d}$ at v3; $1650.42 \pm 642.11 \mathrm{ml} / \mathrm{d}$ at V4 $(p<0.001)$. Total fluid intakes were more important in the beginning of Ramadan than those in the period after Ramadan $(3146.16 \pm 847.01 \mathrm{ml} / \mathrm{d}$ at V2, $2736.47 \pm 801.35 \mathrm{ml} / \mathrm{d}$ at V3 and $2778.76 \pm 738.69 \mathrm{ml} / \mathrm{d}$ at V4 $p<0.001$ ) for type 2 diabetes.

Caloric Intake: Caloric intakes were globally significantly less important during the second period of Ramadan (2416.99 $\pm 471.41 \mathrm{cal} / \mathrm{d}$ inV2, $2214.73 \pm 498.31 \mathrm{cal} / \mathrm{d}$ in $\mathrm{V} 3,2527.15$ $\pm 596.91 \mathrm{cal} / \mathrm{d}$ in V4 p $<0.001$ ).

Carbohydrate intake: Carbohydrate intake were smaller in the second period of Ramadan (v2 : 305.07+/-66.82 g/d, v3: $276.04 \pm 74.92 \mathrm{~g} / \mathrm{d}, \mathrm{v} 4: 319.20 \pm 89.26 \mathrm{~g} / \mathrm{d} p=0.28$ ). Saccharose intakes were significantly higher during the period after the month of Ramadhan $(21.50 \mathrm{~g} / \mathrm{d} v 2,27.86 \mathrm{~g} / \mathrm{d} v 3,28.03 \mathrm{~g} / \mathrm{d} \mathrm{v} 4-p$ $<0.002)$ This was probably linked in the period after Ramadan (of Aid el fatr).

Contributions in proteins: Protein intakes were significantly smaller in the second period of Ramadan (v2 76.73, 70.49 v3, v4 $76.97 p<0.001$ ). However, animal protein intake had no significant changes during the three periods, with an average of around $40 \mathrm{~g} / \mathrm{d}$, and unlike plant proteins whose contributions were significantly smaller in the second period of Ramadan ( v2 $36.41,31.91 \mathrm{v} 3, \mathrm{v} 437.38 \mathrm{~g} / \mathrm{d} p<0.001$ )

Contributions in fat: Intakes of total fat were also significantly smaller in the second period of Ramadan (v2 99.99, $92.56 \mathrm{v} 3$, v4 $101.45 p<0.01$ ). This decrease is observed for saturated fat intake as for polyunsaturated fats

Mono-unsaturated fatty acids are relatively more consumed, representing approximately $50 \%$ of the total amount of fatty acid. Their contributions were also significantly smaller in the second period of Ramadan (49.23 \% v2, v3 $46.55 \%, 50.01 \%$ v4, $p<0.001$ ).

The cholesterol intake was significantly higher during the second half of Ramadhan $(241.76 \mathrm{mg} / \mathrm{d}$ v2, v3 $316.46 \mathrm{mg} / \mathrm{d}$, $207.11 \mathrm{mg} / \mathrm{d}$ v4 $p<0.001$ ).

Contributions minerals, micronutrients and fiber: Calcium intakes were also significantly smaller in the second period of Ramadan (790.59 v2, v3 739.36, $778.36 \mathrm{v} 4 p=0.01)$. It is the same for the intake of sodium, potassium, phosphorus, iron, folic acid, magnesium and vitamins $B$ and $C$, but not for the intake and zinc fibers. The main points regarding the Dietary intake.

This study reveals a significant increase in fluid intake, mainly 


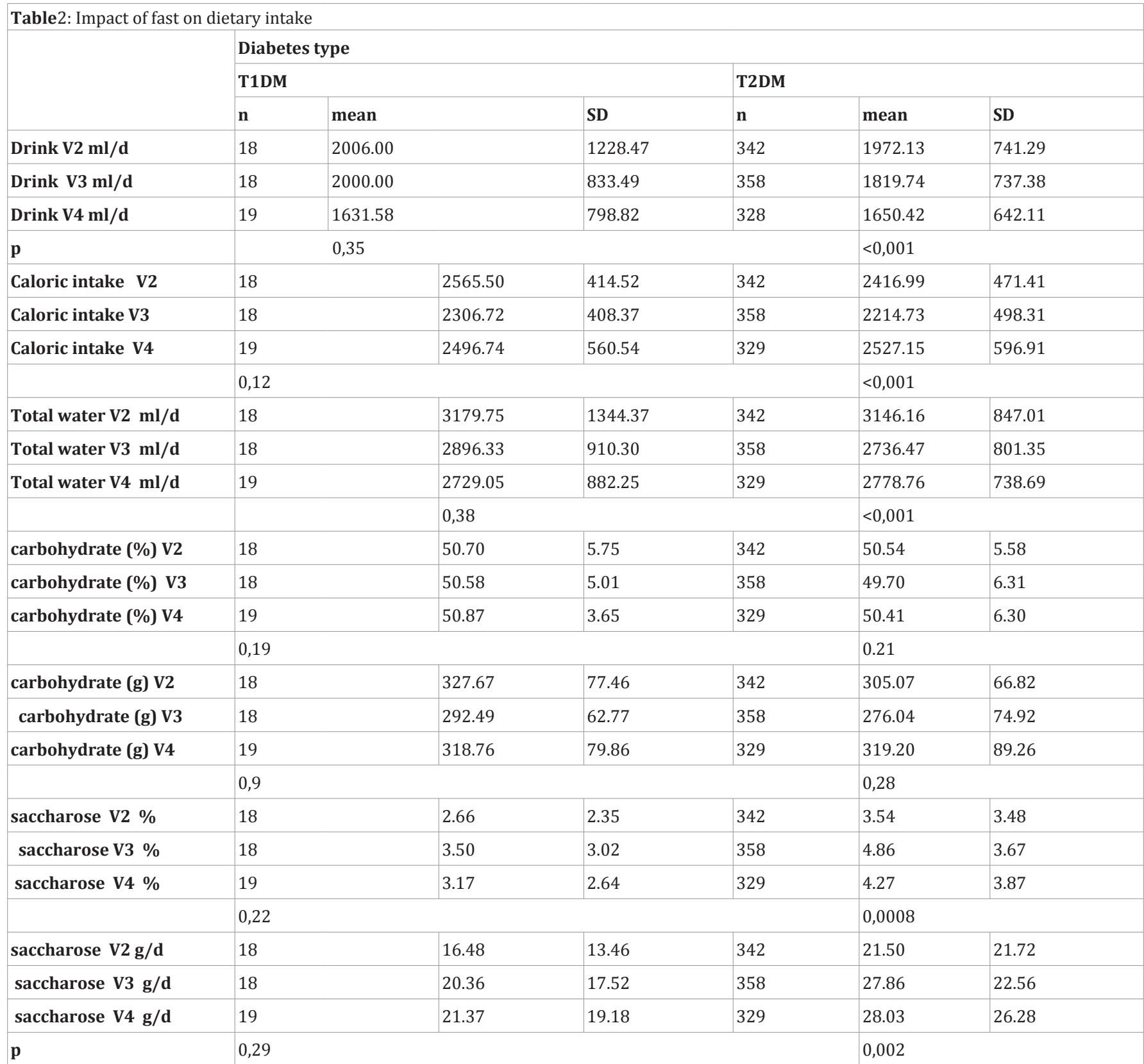

water at the beginning of Ramadan. There was a significant reduction in caloric intake in visit 3 compared to a considerable increase post Ramadan. The supply in carbohydrates are less important during the second period of Ramadan. Whereas contributions in saccharose were significantly more important during the second period as well as after the month of Ramadan The energy intake during Iftar represents more than $90 \%$ of the daily caloric supplies.

\section{Summary of results}

In summary, the largest observational study of fast during the month of Ramadan in Tunisia concerning 533 patients among whom $5.4 \%$ was with type 1 diabetes. Almost $38 \%$ were managed with insulin and half of them practiced a blood sugar monitoring.
During the first and last period of Ramadan, respectively 7.8 and 6.4 of the patients interrupted their fast temporarily. This study proves that fasting patients changed their life style. In fact, the tendency at the beginning of Ramadan is a decrease in physical activity and caloric intake compared to an increase in fluid ingestion. However, the contributions in saccharose are more important during the second half and after Ramadan. No case of severe or nocturnal hypoglycemia was reported. Approximately $24 \%$ of the patients adapted their treatment at the beginning of Ramadan. Glycemic controlled was sustained even improved at the beginning of Ramadan.

\section{Discussion}

Fasting increases the risk of hypoglycemia in patients 
with diabetes, in part related to potentially impaired counterregulatory responses to low blood glucose [1]. Thus, treatment guidelines recommend that most patients with diabetes should not fast during Ramadan [2,3]. However, fasting of Ramadan is observed by a large proportion of Muslims with diabetes. So, in the current study, we proposed to identify the impact of Ramadan fasting on food intake, metabolic parameters and anthropometry.

At the best of the authors' knowledge, this study is the first large observational study which was interested in the details of caloric and drink intake in Ramadan by diabetic patients.

The results showed that the practice of fasting without interruption is relatively common in diabetic persons and the majority of them were type 2 diabetic $(94,6 \%)$. Concerning this group an individual assessment of the situation for each patient is essential, together with patient education and appropriate adjustment of anti-diabetic therapy. These results are in accordance with other epidemiologic study showing that the majority of patients with type 2 diabetes (78.8\%) fasted for at least 15 days during Ramadan [4]. Whereas, according to the other studies, fasting has been uniformly discouraged $b$ by the medical profession for individuals with type 1 diabetes especially those with poor diabetes control because increased risk of hypoglycemia, hyperglycemia and ketoacidosis [5].

Other interesting result was found in this present study, a slight decrease in plasma glucoses' levels during Ramadan fasting in comparison with period of before fasting. As to HbA1C levels the proportion of normal values did not observe significant variations. These results are in line with other studies showing an amelioration of glycemic control in Ramadan [1,6].

Despite slight reduction in the total energy intake, we detected a significant elevation in total weight after fasting. This is in contrast with the literature results, presenting a weight reduction is most often reported and attributed to lower calories intake during Ramadan $[7,8]$.

In non-diabetic individuals, fasting is associated with improvement in several homeostatic risk markers for cardiovascular disease, including reduction in plasma triglyceride and Plasma Low Density Lipoprotein (LDL)-cholesterol level, as well as improvement in insulin sensitivity, leptin, adiponectin and High Density Lipoprotein (HDL) cholesterol [2-5]. Ramadan fasting in non-diabetic individuals is also associated with reduction in plasma homocysteine, D-dimer level, C-Reactive Protein (CRP) and interleuquine 6 and fibrinogen $[7,9]$. Similar beneficial effects of fasting have been reported in diabetic individual. In the current study, we found a trend to decrease in total-Cholesterol and increase in HDL-C. Our results are in accordance with studies on the effect of Ramadan fasting on blood lipids have produced variable results. In fact, it was reported that raised concentrations of cholesterol may be related to weight loss during Ramadan fasting [8]. However, others report have found no change, or only decreased levels of cholesterol during fasting [10]. The evidence that an increase in plasma HDL-C occurs after Ramadan fasting is promising $[11,12]$.
The strength of our study is the dietary investigation. Our finding showed that total water intake is more important at the beginning of Ramadan compared to the period after Ramadan. The calorie intake was significantly lower during the second period of Ramadan. These results able us to hypothesize that this amelioration is linked with an adaptation of the patients in the fast.

The comparison of the macronutrient consumption before and during Ramadan, revealed a significant decrease in fat, in protein and in glucose intake during the second period of Ramadan. However, saccharose intakes were significantly higher during the period after Ramadan. This is probably connected in the consumption of candies and sweets after ramadan

In our study the complications were dominated by the hypoglycemia, indeed decreased food intake is a well-known risk factor for the development of hypoglycemia [6]. The effect of fasting during Ramadan on rates of hypoglycemia in patients with diabetes is not known with certainty. The largest dataset is the EPIDIAR study, which showed that fasting during Ramadan increased the risk of severe hypoglycemia (defined as hospitalization due to hypoglycemia) 4.7 times in patients with type 1 diabetes and 7.5 times in patients with type 2 diabetes [1]. During the present study no severe hypoglycemia was reported.

In a recent five-country observational study, the overall incidence of symptomatic hypoglycemia was $20 \%$ during Ramadan in sulphonylurea-treated Muslims with type 2 diabetes, with a range of $14-26 \%$ with the different sulphonylurea agents [9]. In small clinical trials, similar improvement in glycemic control and a greater incidence of hypoglycemia were observed with gliclazide relative to vildagliptin and with glibenclamide relative to repaglinide during Ramadan [10-12]. Given their widespread use and potential for adverse effects, there is a need for additional clinical studies evaluating the effects of sulphonylurea compared with alternative treatment options in patients who fast during Ramadan.

Sitagliptin, a dipeptidyl peptidase-4 (DPP-4) inhibitor, has been shown to be effective and well tolerated with a low incidence of hypoglycemia in clinical trials up to 2 years in duration [13]. When added to ongoing metformin mono-therapy, the addition of sitagliptin was shown to reduce the incidence of symptomatic hypoglycemia, three to sixfolds, compared with the addition of a sulphonylurea in patients with T2DM [9].

In our study, we didn't use the DPP4 inhibitors therapy because they were not available in our country in the period of the study. In recent years, exciting new therapies and technologies have positively influenced the management of diabetes. Some of these have been investigated for use during the fasting of Ramadan. The DPP-4 inhibitors vildagliptin and sitagliptin provide an effective and safe therapeutic option during Ramadan, either alone or in combination with metformin or sulfonylureas. The incritinmimetics liraglutide and exenatide are potentially safe therapies during Ramadan, but as yet there are no reports of using them during Ramadan. Recently, glycemic therapeutic options for diabetes have expanded, with the introduction of new 
therapeutic agents and new technologies; some of these have been used during Ramadan and have shown potential therapeutic benefit further clinical trials are needed to evaluate the safety and efficacy of new anti-diabetic agents and new diabetes-related technologies during Ramadan.

However, the practical management of diabetes in Ramadan is made on an individual basis; newer pharmacological agents have lesser hypoglycemic potential and may have specific advantages. Similarly, insulin pump therapy may provide greater safety in the Ramadan setting [1]. In our study, treatment based dual therapy was observed in $(31,1 \%)$ of cases and monotherapy in $(22,8 \%)$ of cases, the main families are represented by biguanides $(44,5 \%)$ followed by sulfonylureas $(39,2 \%)$. The main types of insulin is long acting analog Insulin (51.9\%) followed by unspecified insulin $(19,1 \%)$.

The newer sulfonylureas gliclazide MR and glimepride can be safely used during Ramadan, but glibenclamide should be avoided because of the increased risk of hypoglycemia. Administration of the long-acting insulin (glargine and detemir); or the premixed insulin analogues has shown potential benefit in selected patients with T1DM and T2DM diabetes. The insulin pump can potentially empower patients with diabetes to safe fasting during the month of Ramadan. Clinical trials are needed in order to further evaluate the safety and efficacy of new hypoglycemic agents and new diabetes-treating technologies during Ramadan.

Finally a review of available medical literature indicates that fasting in Ramadan is safe and probably beneficial with proper education and good management for the majority of persons suffering from diabetes [14]. This is so especially for type 2 diabetics. Healthy, stable and well informed type-1 diabetics are also able to fast safely but need to be supervised and managed with greater care and strict attention to their diet, daily activities, glycemic control, and insulin dosage adjustments. So that, Careful and individual evaluation and open discussions are key measures for ensuring patient safety during the fasting of Ramadan [15]. The physician is duty-bound to support diabetic individuals before and through Ramadan to enable safe fasting. Patients who insist on fasting need to be aware of the associated risks and be ready to adhere to the recommendations of their healthcare providers to achieve a safer fasting experience.

\section{Conclusion}

As a conclusion, in this real life study we observe a good tolerability and safety of fasting Ramadan in diabetic patients. Recently, glycemic therapeutic options for diabetes have expanded, with the introduction of new therapeutic agents and new technologies. Some of these have been used during Ramadan and have shown potential therapeutic benefit. Further clinical trials are needed to evaluate the safety and efficacy of new antidiabetic agents and new diabetes-related technologies during Ramadan. Furthermore, patients who insist on fasting need to adhere to the recommendations of their healthcare providers to achieve a safer fasting experience.

\section{Acknowledgements}

The authors thank Sanofi Laboratories for sponsoring this work as well as all the investigators who have to display huge efforts important for the finalization of this study. They are: Tarzi Hichem; Sellami Samia; Nagati Khemaies; Mekawer Awatef; Kharrat Selwa; Jelouli Kamel; Jait Faycal; Ennabli Nawar Meriem; Doghri Taieb; Derouiche Fethi; Cheraga Leila; Chammakhi Saïd; Bouguerra Radhia; Aissaoui Mohamed; Ben Mami Feyka; Mestiri Akil; Gaigi Sadok; Blouza Samira; Turki Zinet; Ben Abdallah Nejib; Zidi Borni; Zrig Nebila; Achour Ahmed; Ben Slama Claude; Trimeche Rached, Abid Abdelmajid; Hamzaoui Jazia; Marouani Oueslati Awatef; Fetouhi Bochra; Jamoussi Henda; Jaballah Ibtissem; Ernez Salambo; Ardhaoui Ilhem; Maaroufi Amel; Chaieb Elarbi; Chaieb Molka; Trimeche Sihem; Kacem Maha; Sfar Habib; Karray Foued; Kammoun Kamel; Kammoun Selma; Kaffel Noureddine; Kachouri Moncef; Bellaswed Med; Abbes Wissal; Abid Mohamed; Belaid Jamil;Garali Mounir; Derbel Adnene; Khochtali Ines; Miladi Riadh; Atallah Mohamed; Ouertani Mohamed; Cherif Lotfi; Ben Brahim Samir; Ben Rhayem Boutheina

\section{References}

1. Salti I, Benard E, Detournay B, Bianchi-Biscay M, Le BC, Voinet C, et al. A population-based study of diabetes and its characteristics during the fasting month of Ramadan in 13 countries: results of the epidemiology of diabetes and Ramadan 1422/2001 (EPIDIAR) study. Diabetes Care. 2004;27(10):2306-11.

2. Khaled BM, Belbraouet S. Effect of Ramadan fasting on anthropometric parameters and food consumption in 276 type 2 diabetic obese women. Int J Diabetes Dev Ctries. 2009;29(2): 62-68.

3. Suliman M, Abdu T, Elhadd T, Ibrahim S, Ahmed MH, Malik R. Diabetes and fasting in Ramadan: Can we provide evidence-based advice to patients? Sudan Med J. 2010;46(1):4-14.

4. Sari R, Balci MK, Akbas SH, Avci B. The effects of diet, sulfonylurea, and Repaglinide therapy on clinical and metabolic parameters in type 2 diabetic patients during Ramadan. Endocr Res. 2004;30(2):169-77.

5. Schernthaner G, Grimaldi A, Di MU, Drzewoski J, Kempler P, Kvapil M, et al. GUIDE study: double-blind comparison of once-daily gliclazide MR and glimepiride in type 2 diabetic patients. Eur J Clin Invest. 2004;34(8):535-42.

6. Sadiya A, Ahmed S, Siddieg HH, Babas IJ, Carlsson M. Effect of Ramadan fasting on metabolic markers, body composition, and dietary intake in Emiratis of Ajman (UAE) with metabolic syndrome. Diabetes Metab Syndr Obes. 2011;4:409-16.

7. Al-Arouj M, Bouguerra R, Buse J, Hafez S, Hassanein M, Ibrahim MA, et al. Recommendations for management of diabetes during Ramadan. Diabetes Care 2005;28:2305-2311.

8. Bravis V, Hui E, Salih S, Mehar S, Hassanein M, Devendra D. Ramadan Education and Awareness in Diabetes (READ) programme for Muslims with type 2 diabetes who fast during Ramadan. Diabet Med. 2010;27(3):327-31.

9. The effect of intensive treatment of diabetes on the development and progression of long-term complications in insulin-dependent diabetes mellitus. The Diabetes Control and Complications Trial Research Group. N Engl J Med. 1993;329(14):977-86.

10. Hallak MH, Nomani MZA. Body weight loss and changes in blood lipid level in normal men on hypocaloric diets during Ramadan fasting. Am J Clin Nutr. 1988;48(5):1197-210.

11. Maislos M, Khamaysi N, Assali A, Abou-Rabiah Y, Zvili I, Shany S. Marked increase in plasma high-density lipoprotein cholesterol after prolonged fasting during Ramadan. Am J Clin Nutr. 1993;57(5):640-2. 
12. Temizhan A, Tandogan I, Donderici 0, et al. The effects of Ramadan fasting on blood lipid levels. Am J Med. 2000;109(4):341-2.

13. Wissam HI, Hosam MH, Amjad HJ, Samer AAB. Effect of Ramadan fasting on markers of oxidative stress and serum biochemical markers of cellular damage in healthy subjects. Ann Nutr Metab. 2008;53(34):175-81.
14. Mohammed Abdul Jaleel, Syed Abbas Raza, Farah Naaz Fathima, BushraNaazFathima Jaleel. Ramadan and diabetes: As-Saum (The fasting). Indian J Endocrinol Metab. 2011;15(4):268-73.

15. Effects of Fasting Ramadan on health and athletic performance .Dr. Hamdi Chtourou. OMICS Group International. 2015. 